\title{
Topological analysis of paraxially scattered electron vortex beams
}

\author{
Axel Lubk, ${ }^{1,2}$ Laura Clark, ${ }^{2}$ Giulio Guzzinati, ${ }^{2}$ and Jo Verbeeck ${ }^{2}$ \\ ${ }^{1}$ Triebenberg Laboratory, Institute of Structure Physics, Technische Universität Dresden, 01062 Dresden, Germany \\ ${ }^{2}$ EMAT, University of Antwerp, Groenenborgerlaan 171, 2020 Antwerp, Belgium
}

(Received 3 January 2013; published 27 March 2013)

\begin{abstract}
We investigate topological aspects of subnanometer electron vortex beams upon elastic propagation through atomic scattering potentials. Two main aspects can be distinguished: (i) significantly reduced delocalization compared to a similar nonvortex beam if the beam centers on an atomic column and (ii) site symmetry dependent splitting of higher-order vortex beams. Furthermore, the results provide insight into the complex vortex line fabric within the elastically scattered wave containing characteristic vortex loops predominantly attached to atomic columns and characteristic twists of vortex lines around atomic columns.
\end{abstract}

DOI: 10.1103/PhysRevA.87.033834

\section{INTRODUCTION}

Electron vortex beams carrying orbital angular momentum (OAM) recently gained considerable interest after the experimental demonstration of so-called vortex electron beams in a transmission electron microscope (TEM) [1,2]. Their properties were predicted earlier as an extension to optical vortices, including their charged particle character [3]. Similar to optical vortices $[4,5]$ several applications have been demonstrated, but especially the ability to focus electron vortex beams to the atomic scale [6,7] and their strong interaction with matter $[8,9]$ holds promise for atomic resolution mapping of magnetic states in materials by means of electron magnetic circular dichroism (EMCD).

The linear wave equations governing the motion of the electron beam allow eigensolutions to arbitrary OAM [10,11], which are experimentally created by dedicated apertures in TEM $[1,2,6]$. Such beams must contain a phase singularity, which possesses a more basic structure only loosely connected to OAM [12]: Topological concepts can be used to precisely define that singularity in terms of topological charges, thereby characterizing the electron wave [13]. However, for the particular case of highly localized electron vortex beams scattering at atomic potentials almost nothing is known about their vortex behavior upon propagation. We will apply both (scalar) electron wave dynamics and topological concepts in order establish conservation laws and to study the interaction between vortex lines and electrostatic potentials. This topological information is usually too coarse for quantitative computation of, e.g., scattering cross sections of core losses in energy electron loss spectroscopy (EELS). However, it provides qualitative understanding and concepts sometimes without solving the scattering problem itself. For example, we will show subsequently that wave topology is sufficient to characterize some aspects of the wave function upon propagation, such as the creation and destruction of electron vortex beams, the splitting of higher-order vortex beams, etc. We also stress the generality and beauty of topological concepts by appealing to the visual perception, often providing insights via simple graphs.

The paper is organized in the following way. In the next section, we introduce the topological concepts, i.e., a suitable wave-function topology including its invariant, which are used later on. Subsequently we will briefly introduce the scalar paraxial wave equation, which facilitates a highly accurate
PACS number(s): 41.85.-p, 42.50.Tx, 41.20.Jb, 41.75.Fr

description of the dynamics of accelerated electron waves. Finally we combine wave dynamics and topology in order to characterize the vortex structure of the wave field and its interaction with atomic scattering potentials.

\section{TOPOLOGICAL INVARIANTS OF THE (VORTEX) WAVE}

Topological invariants are features that do not change upon continuous transformation of the wave field. Since we restrict our considerations to the paraxial regime, that transformation is the propagation along the optical axis $z$ and we shall focus on the topology and its invariants of two-dimensional (2D) wave functions defined on planes with Cartesian coordinates $\boldsymbol{r}_{\perp}=(x, y)^{T}$ (polar: $r, \theta$ ) perpendicular to $z$. Generally, the $2 \mathrm{D}$ wave function is an assignment $\Psi: \mathbb{R}^{2} \rightarrow \mathbb{C},(x, y) \rightarrow$ $\Psi(x, y)$. Such a map can be considered as a vector field $(\operatorname{Re}\{\Psi\}, \operatorname{Im}\{\Psi\})^{T}$ composed from real and imaginary components of the complex wave. The field might then contain zero-dimensional (OD) vortex (or phase) singularities where the phase vorticity of the phase vector field diverges [e.g., Fig. 1(a), see Appendix A for details]. In this work we will use domain coloring (e.g., [14]), i.e., mapping of the wave function $\Psi=|\Psi| \exp (i \varphi)$ into HSV (hue, saturation, value) color space (amplitude $|\Psi| \rightarrow \mathrm{V}$, phase $\varphi \rightarrow \mathrm{H}$ ), to solve the nontrivial visualization problem for the wave function [Fig. 1(b); in the grayscale print version green appears as light gray, blue as dark gray with red in between]. In such maps, phase singularities show up as prominent color wheels elegantly revealing the $2 \pi$ phase jumps inherent to vortices.

The phase singularities visible in Fig. 1 are characterized by nonvanishing integer winding numbers $w \in \mathbb{Z}$ of the phase gradient integrated along certain paths $2 \pi w=\oint d s \cdot \nabla \varphi(d s$ is the path element). For example. $w$ either assumes $m$ (path I) or 0 (path II) in Fig. 1(b) and paths yielding the same $w$ are equivalent under homotopy (i.e., can be continuously transformed into each other, e.g., [15]). By applying Stokes theorem

$$
2 \pi w=\iint_{S} d^{2} r_{\perp}(\nabla \times \nabla \varphi) \cdot \boldsymbol{n}=\iint_{S} d^{2} r_{\perp} \boldsymbol{\Theta}_{p} \cdot \boldsymbol{n},
$$

where $S$ is the enclosed area with unit normal $\boldsymbol{n}$, one can define the phase vorticity distribution $\boldsymbol{\Theta}_{p}$ which is zero everywhere except at $0 \mathrm{D}$ phase singularities. We will use this definition to numerically locate vortices in the wave field later on. We now define the total winding number $W$ as the phase 

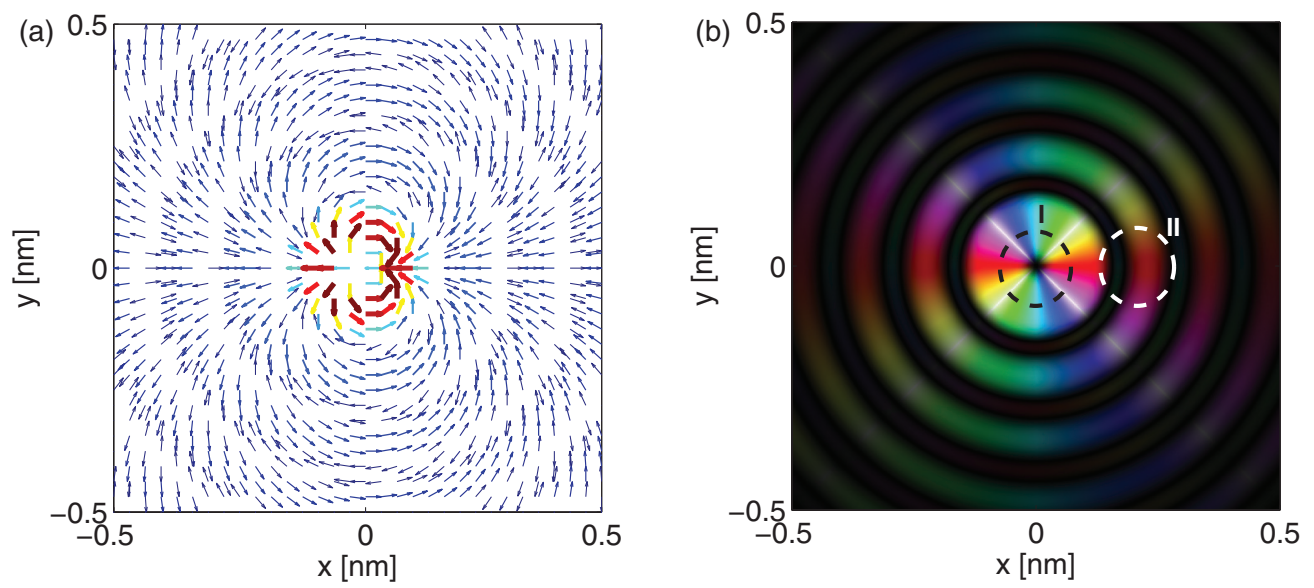

FIG. 1. (Color online) (a) Polya map of vortex beam $\Psi_{m=2}$ [see Eq. (13) for details]. Amplitude of the complex number is color coded (light blue and thin line $\cong$ small, dark red and thick line $\cong$ large). The Kronecker (Hopf) index of the phase singularity equals $m$ and corresponds to the winding number $w$ of any closed path around the singularity. (b) Amplitude (value) and phase (hue) of the same $\Psi_{m=2}$ encoded as a HSV image (domain coloring, in the print version green $\cong$ light gray, blue $\cong$ dark gray with red in between). The fully saturated white lines are used to display constant phase lines which end at the phase singularity by definition.

vorticity integrated over the whole plane, which can also be computed from the sum of path integrals $j$ containing exactly one of the $N$ vortices, i.e.,

$$
W=\iint d^{2} r_{\perp} \boldsymbol{\Theta}_{p} \cdot \boldsymbol{n}=\sum_{j=1}^{N} w_{j}
$$

(e.g., [15]). $W$ is a topological invariant constant under propagation because it is a continuous assignment restricted to integer values (for an alternative derivation of the conservation of $W$ see below; elaborate proofs can be found in the literature [15]). We furthermore note two related facts about the wave function in the vicinity of a vortex core at $\left(x_{j}, y_{j}\right)^{T}$ [with local coordinates $r_{j}=\sqrt{\left(x-x_{j}\right)^{2}+\left(y-y_{j}\right)^{2}}$ and $\theta_{j}=$ $\left.\arctan 2\left(y-y_{j}, x-x_{j}\right)\right]:(1) \Psi \sim \exp \left(\operatorname{im} \theta_{j}\right)$ which coincides with the azimuthal behavior of a spherical harmonic with angular momentum pointing along $z: Y_{l \pm l} \sim e^{ \pm i l \theta_{j}}$. That leads to object entangled inelastic excitation of $\Psi \sim \exp [i(m \pm$ $\left.0,1) \theta_{j}\right]$, which provides a suitable basis for measuring EMCD with OAM beams $[1,8]$. (2) The angular momentum

$$
\begin{aligned}
-i \hbar \int d^{2} r_{\perp} \Psi^{*} \partial_{\theta_{j}} \Psi & =\hbar \int d r_{j} r_{j} \int_{0}^{2 \pi} d \theta_{j}|\Psi|^{2} \partial_{\theta_{j}} \varphi \\
& =\hbar \int d r_{j} r_{j} \oint d \boldsymbol{s} \cdot \nabla \varphi|\Psi|^{2}
\end{aligned}
$$

around a phase singularity has the same sign as the winding number if the gradient of the phase along the circular path does not change sign. That facilitates a prediction of the direction of the angular momentum close to the vortex.

We will now briefly review the behavior of vortices upon small perturbations (without vortex signature) since this will reveal a classification of possible continuous deformations of the vortex beam during propagation along $z$ (similar considerations can be found in Ref. [13]). For that purpose it is sufficient to consider the lowest-order Taylor expansion of the wave around the phase singularity given by $\Psi\left(\boldsymbol{r}_{\perp}, z=\right.$ $0)=(x+i y)^{m} \sim \exp (\operatorname{im} \theta)$ [generally, an $O(m)$ expansion is required to describe an $m$-order vortex]. We begin with the most simple perturbation consisting of adding a linear perturbation function $(a x+b z+c$ with $a, b, c \in \mathbb{R}$ without loss of generality) to a $m=1$ vortex. In the limit of small $a, b$, and $c$ (continuous transformation),

$$
\Psi^{\prime}(\boldsymbol{r})=(x+i y)+a x+b z+c+O\left(\boldsymbol{r}^{2}\right) .
$$

Accordingly, the singularity $\Psi^{\prime}\left(x_{0}, y_{0}\right)=0$ gets shifted to

$$
x_{0}=-(b z+c) /(1+a) .
$$

A first-order Taylor expansion around $x_{0}, y_{0}=0, z_{0}=0$ yields

$$
\Psi^{\prime}(\boldsymbol{r})=(1+a) x^{\prime}+i y^{\prime}+b z^{\prime}=x^{\prime \prime}+i y^{\prime},
$$

where a rotation around the $y$ axis with an angle $\alpha=$ $\arctan \frac{b}{1+a}$ was performed in the last step. Consequently, a small perturbation added to a $m=1$ beam can only continuously shift and tilt the vortex $\cong$ the vortex is topologically protected and the loci of phase singularities in three dimensions form a line). It is interesting to note that there is no restriction in the rotation angle $\alpha$, e.g., a $90^{\circ}$ tilt of the vortex to the side, i.e., $\Psi(\boldsymbol{r})=x+i z+O\left(\boldsymbol{r}^{2}\right)$, is possible through continuous perturbations. The situation becomes slightly more complex at higher orders of $m$ due to the following effect: Adding a small constant to the vortex, i.e., $(x+i y)^{m}+c$, will lift the degeneracy of the zero, leading to $m$ complex zeros, i.e., the $m>1$ beam will be transformed into $m$ first-order beams, which will then follow the perturbation rules developed above. Since the requirement for splitting of a higher-order vortex beam is weak (small $c$ ) we do not observe stable higher-order beams in the experiment unless symmetry of the scattering potential prevents the $m$-fold splitting. In the light of the last discussion, a higher-order vortex can be regarded as a degenerate superposition of nondegenerate first-order vortices where the details of degeneracy lifting can be analyzed by topological arguments [16]. Similarly, also noncollinear crossing of vortex lines, e.g., $\Psi(\boldsymbol{r})=(x+i z)(y+i z)+O\left(\boldsymbol{r}^{3}\right)$, can occur in the wave field. In total, we can identify three main effects upon perturbation: shift, tilt, and (non-)collinear crossing, all of which can occur upon paraxial propagation of the wave, and 
we will show in Sec. III that the simple perturbation scheme discussed above is indeed sufficient to describe the possible modulation of vortices. Note that none of the above effects destroys a vortex line similar to the second Helmholtz theorem in fluid mechanics [17]. Consequently also the total number of vortices $W$ remains constant corroborating the proof sketch above. By evaluating the location of the vortex loci upon propagation we can detect the vortex line skeleton, hence the topological structure of the wave function.

\section{PARAXIAL WAVE DYNAMICS}

The scalar paraxial wave equation governing the propagation of a fast electron wave inside a TEM reads [18 ${ }^{1}$

$$
\partial_{z} \Phi(\boldsymbol{r})=i\left(\frac{E V(\boldsymbol{r})}{k_{0} \hbar^{2} c^{2}}+\frac{1}{2 k_{0}} \Delta_{\perp}\right) \Phi(\boldsymbol{r}),
$$

and thus can be considered as a time-dependent 2D Schrödinger equation $\left(k_{0}\right.$ is the magnitude of wave vector, $E$ is the energy of electron wave, and $V$ is the electrostatic scattering potential). Consequently, we can analyze commutator relations of certain operators with the Hamilton operator, determining whether they are conserved or not upon propagation. In the case of OAM the commutator $\left[L_{z}, H\right]=\left[-i \hbar \partial_{\theta}, V\right]$ only vanishes if $V$ does not depend on $\theta$, i.e., OAM is not conserved upon propagation (contrary to the total winding number) and can even change sign (see [19] for explicit numerical calculation). This can be also understood by noting the paraxial equation in the rotating basis $\Phi(\boldsymbol{r})=\chi(\boldsymbol{r}) \exp (\operatorname{im} \theta)$;

$$
\partial_{z} \chi(\boldsymbol{r})=i\left(\frac{E V(\boldsymbol{r})}{k_{0} \hbar^{2} c^{2}}+\frac{1}{2 k_{0}} \Delta_{\perp}-\frac{m^{2}}{2 k_{0 z} r^{2}}+\frac{i m}{k_{0} \hbar r^{2}} \hat{L}_{z}\right) \chi(\boldsymbol{r}),
$$

where a nonvanishing last term leads to a destruction of the initial OAM eigenstate. The propagation of an electron wave along $z$ can be approximated by successive free space Fresnel propagation $\Phi\left(\boldsymbol{r}_{\perp}, z+\delta z\right)=\left[1+i \delta z /\left(2 k_{0}\right) \Delta_{\perp}\right] \Phi\left(\boldsymbol{r}_{\perp}, z\right)$ and projected potential phase shifts $\Phi\left(\boldsymbol{r}_{\perp}, z+\delta z\right)=[1+$ $\left.i C_{E} V_{p}\left(\boldsymbol{r}_{\perp}\right)\right] \Phi\left(\boldsymbol{r}_{\perp}, z\right)\left[C_{E}=\frac{E}{k_{0} \hbar^{2} c^{2}}, V_{p}\left(\boldsymbol{r}_{\perp}\right)=\int_{z}^{z+\delta z} d z V(\boldsymbol{r})\right]$ for small propagation steps $\delta z$. The corresponding numeric integration scheme is referred to as multislice [20] and will be used below.

We will now show that the previously identified generic situations (i.e., shift, bending, splitting) are locally compatible with the paraxial equation, which will effectively impose certain constraints on the perturbation parameters $a, b$, and $c$. Since solutions with vortices oriented along $z$ are well known it remains to discuss the horizontal orientation of vortex lines. Indeed, the horizontal vortex line with the local shape

$$
\Psi\left(\boldsymbol{r}_{\perp}, z=0\right)=x+2 k_{0}\left(a x^{2}+b y^{2}\right)+O\left(r^{3}\right),
$$

and second-order term coefficients $a+b=1$ lead to the required $i z$ dependency when propagated at small distances. The superposition of perpendicular (noncollinear) vortex lines, e.g., the combination of two vortex lines oriented along $y$ and

\footnotetext{
${ }^{1}$ Shortcomings of the paraxial approximation as well as vector potentials will be considered elsewhere.
}

$x$ to $\Psi(\boldsymbol{r})=(x+i z)(y+i z)+O\left(\boldsymbol{r}^{3}\right)$ is also compatible with the paraxial equation if the wave function

$\Psi\left(\boldsymbol{r}_{\perp}, z=0\right)=x y+2 k_{0}\left(a x^{3}+b x^{2} y+c y^{2} x+d y^{3}\right)+O\left(\boldsymbol{r}^{4}\right)$

with $6 a+2 c=1$ and $6 d+2 b=1$. The latter result shows that noncollinear crossing of vortex lines is not forbidden in a paraxial wave field.

We will now analyze the motion of a vortex (see Ref. [21] for similar considerations for optical vortices): In line with the integration scheme noted above we distinguish between (A) phase modulations such as produced by a weak atomic potential $\Phi=\left(1+i C_{E} V_{p}\right)(x+i y)$ and (B) amplitude modulations as occurring under free space propagation ( $\cong$ interference) $\Phi=(1+A x)(x+i y)$. The infinitesimal paraxial free space propagation step [see Eq. (7)] after phase modulation reads

$$
\begin{aligned}
\Phi\left(\boldsymbol{r}_{\perp}, z+\delta z\right) \\
=\left(1+i \frac{\delta z}{2 k_{0}} \Delta\right)\left[1+i C_{E} V_{p}\left(\boldsymbol{r}_{\perp}\right)\right](x+i y) \\
=\left[1+i C_{E} V_{p}\left(\boldsymbol{r}_{\perp}\right)-\frac{\delta z}{2 k_{0}} C_{E} \Delta_{\perp} V_{p}\left(\boldsymbol{r}_{\perp}\right)\right](x+i y) \\
-\frac{\delta z}{k_{0}}\left(\partial_{x}+i \partial_{y}\right) V_{p}\left(\boldsymbol{r}_{\perp}\right)
\end{aligned}
$$

and we recognize a $x(y)$ shift proportional to $\partial_{x} V_{p}\left(\partial_{y} V_{p}\right)$ [see Eq. (3)]. Thus, if the vortex is placed in the vicinity of a radial atomic potential it is attracted towards the positive screened Coulomb potential of an atom. In case of an amplitude modulation one obtains

$$
\begin{aligned}
\Phi\left(\boldsymbol{r}_{\perp}, z+\delta z\right) & =\left(1+i \frac{\delta z}{2 k_{0}} \Delta\right)(1+A x)(x+i y) \\
& =(1+A x)(x+i y)+i \frac{\delta z}{2 k_{0}} A,
\end{aligned}
$$

i.e., the vortex is shifted perpendicular to the modulation direction which will superimpose on phase modulation to yield the total motion of the vortex line. Explicit examples of that behavior will be shown by numerically propagating a vortex wave through a model scattering potential. We have deliberately picked a $\mathrm{SrTiO}_{3}$ single crystal because it facilitates the simulation of a variety of scattering situations due to the combination of heavy and light atomic species in a relatively simple perovskite lattice. Furthermore, the material resembles a large class of perovskite oxides with, e.g., interesting (multi-)ferroic properties which might be probed by EMCD experiments utilizing vortex beams in the near future. The crystal was oriented in the [001] direction to provide well-aligned atomic columns. An incoming vortex beam of order $m$ as produced by the commonly used fork aperture in the condenser aperture of a TEM in scanning mode (STEM) has the general shape of a generalized hypergeometric function

$$
\begin{aligned}
\Phi_{m}\left(r_{\perp}, \theta, z=0\right)= & e^{i m \theta} \int_{0}^{\alpha} J_{m}(k r) k d k \\
= & e^{i m \theta} \frac{2^{-m} r_{\perp}^{m} k_{\max }^{2+m}}{(2+m) \Gamma(1+m)}{ }_{1} F_{2}\left(1+\frac{m}{2} ; 2\right. \\
& \left.+\frac{m}{2}, 1+m ;-\frac{1}{4} r_{\perp}^{m} k_{\max }^{2}\right)
\end{aligned}
$$




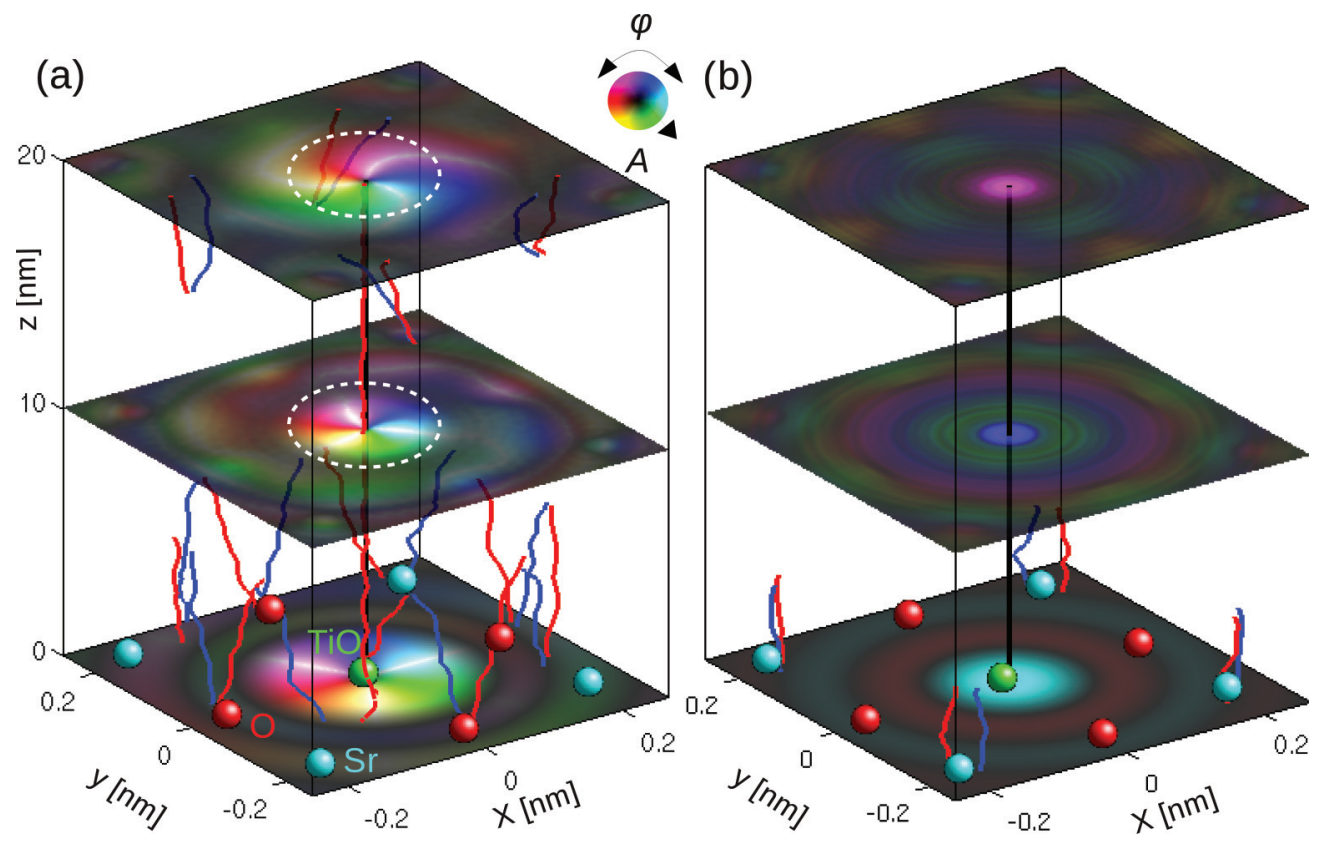

FIG. 2. (Color online) (a) $m=1$ vortex and (b) $m=0$ conventional beam. The wave functions at different $z$ planes are displayed domain colored. $m=1(-1)$ vortex lines are blue (dark gray) [red (light gray)]. Center-of-mass line is black. The atomic columns of SrTiO ${ }_{3}$ are indicated at the bottom plane. The undisturbed vortex region is indicated by a white circle in (a). Note the closed zigzag ring formed by red (light gray) and blue (dark gray) lines at the bottom of (a).

when focused on the crystal entrance face $\left(k_{\max }=k_{0} \alpha_{\max }\right.$ is the aperture size; see Appendix $\mathrm{C}$ for details). In order to facilitate a straightforward discussion of the characteristic vortex propagation features, we restricted our analysis to $m=1,2,4$ vortex beams exactly centered on and slightly off-center $(0.04 \mathrm{~nm})$ particular positions, namely, the $\mathrm{TiO}$ column and the $\mathrm{O}$ column. ${ }^{2}$ Off-centering was investigated in order to consider realistic experimental disturbance of the beam position introduced by instrument limitations, specimen drift, and thermal motion. Parameters of the simulation separated into microscopical (A), material (B), and numerical (C) are (A) $200 \mathrm{kV}$ acceleration voltage, $15 \mathrm{mrad}$ illumination aperture; (B) $\mathrm{SrTiO}_{3}$ (space group $P m \overline{3} m, a=0.395 \mathrm{~nm}$ ), [001] orientation, $20 \mathrm{~nm}$ thickness; and (C) supercell size to suppress artifacts from periodic boundary conditions: $7 \times 7 a \cong$ $(2.765 \times 2.765 \mathrm{~nm}), 560 \times 560$ sampling, $\delta z=0.1975 \mathrm{~nm}$ propagation step, atomic scattering potentials from Ref. [22]. The domain-colored wave will be depicted at different $z$ coordinates and vortex lines will be drawn to illustrate the motion of phase singularities. The vortex lines have been determined by detecting singularities in the phase vorticity [Eq. (1)] and connecting adjacent detected singularities along $z$. The numerical accuracy of the vortex detection is limited by sampling in both the horizontal $\left(r_{\perp}\right)$ and vertical $(\delta z)$ domains, which becomes particularly apparent at the turning points of the loops which are frequently not completely resolved.

In Fig. 2(a) we show the vortex line of a $m=1$ beam centered on the TiO column. The central vortex line is straight and one can distinguish two regions around the vortex: Within

\footnotetext{
${ }^{2} \mathrm{The}$ Sr column results resemble that of the TiO column due to the similar scattering power.
}

the white circle the wave function corresponds well to an angular momentum eigenstate $\sim \exp (\operatorname{im} \theta)$. Outside, the equiphase lines are strongly bent, which coarsely resembles a Rankine vortex behavior [23]. The radius of the circle is determined by strong deviations from the rotationally symmetric scattering potential given by neighboring atomic columns, i.e., the last term of the rotated paraxial Eq. (8) remains small only within the radius. To compare the delocalization upon scattering of vortex and nonvortex (conventional) beams we plot the mean radius $\bar{r}(z)=\left[\int d^{2} r_{\perp} \Phi^{*}(\boldsymbol{r}) r_{\perp}^{2} \Phi(\boldsymbol{r})\right]^{1 / 2}$ of various beams in Fig. 3. Here we note that the presence of a stable topologically protected zero centered on the strongly scattering atomic column prevents the vortex beam from scattering (and delocalizing) rapidly. In the example shown the mean radius even increases less slowly than the freely propagating beam due to the attracting force of the positive atom cores in the column. That behavior stays in contrast to the corresponding (i.e., with same convergence angle) conventional $m=0$ beam [Fig. 2(b)], which initially has his maximum at the column. At the same convergence angle the conventional beam is initially slightly more localized than the vortex beam but delocalizes significantly stronger upon scattering, both compared to the vortex and the freely propagating conventional beam. We remark that the quantitative details of the delocalization reduction of the vortex beam depend on a large set of parameters, such as acceleration voltage, convergence angle, scattering potential, specimen thickness, and hence have to be worked out for each particular problem in practice.

We furthermore observe a formation of numerous vertical vortex loops upon propagation, visible as vortex-antivortex pairs in a particular $z$ plane. They are predominantly attached to atomic columns and have been known for a long time to occur in conventional beams scattered at atomic potentials 


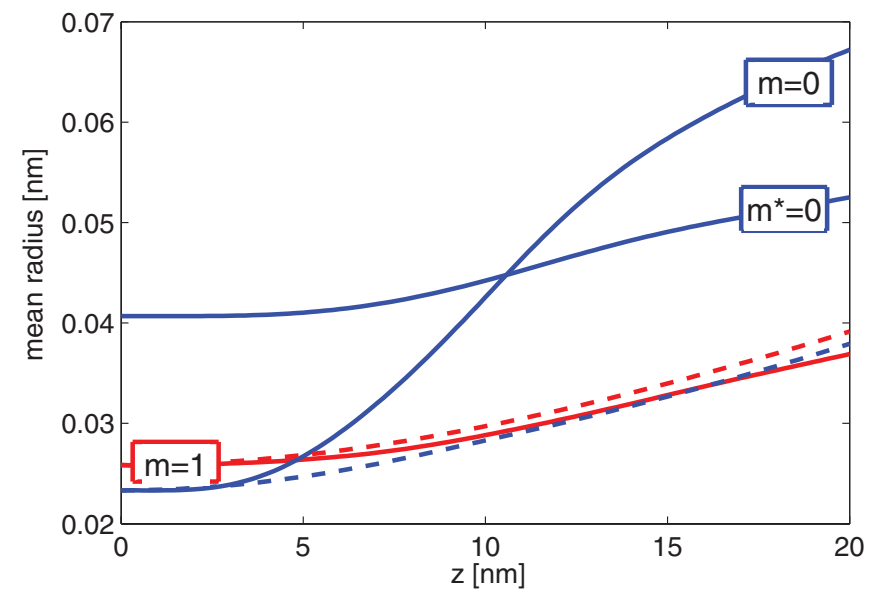

FIG. 3. (Color online) Delocalization upon propagation of STEM beams of two $m=0$ [blue (dark gray); for comparison $m^{*}$ had a strongly reduced opening angle (i.e., larger initial delocalization) of $5 \mathrm{mrad}$ ] and one $m=1$ [red (light gray)] centered on the TiO column. Dashed curves of free propagating beams (without any scattering potential) are shown to illustrate the delocalization effect due to scattering. The $m=1$ vortex beam shows a significantly reduced delocalization compared to the normal $m=0$ beam with the same convergence angle.

[Fig. 2(b)] [24]. Their presence visually explains the local sign flips in the local angular momentum density [see Eq. (3)], observed in Ref. [19]. Less frequently we also observed predominantly horizontally oriented loops. For example, the vortex-antivortex pairs emerging from the oxygen column in Fig. 2(a) form a horizontal zigzag loop enclosing the central vortex. However, we could not detect any knot structure (i.e., linked loops) in the wave field as reported for photonic wave fields [25]. Thus they seem to be a less probable structure although they are not forbidden in general.
Besides vortex loops the off-center $m=1$ beam [Fig. 4(a)] also shows circulation of the central singularity around the TiO column. That circular motion is the sum of a phase modulation attracting the vortex towards the atomic column and an amplitude modulation (focusing property of positive atomic potential) forcing an angular shift (see above). It is not observed when tracking the center of mass [see Fig. 4(a)]. In agreement with Eq. (5) the spiral direction changes when the topological charge changes sign. If the beam is centered on the $\mathrm{O}$ column the circulation period is increased as a consequence of the smaller scattering potential of the atomic column [Fig. 4(b)].

In the case of higher-order beams $m>1$ we additionally expect to observe splitting into $m$ first-order beams (see above). We found a restriction to this behavior depending on the site symmetry around the beam: At positions without rotational symmetry the higher-order beams split into $m$ first-order beams. At positions with local $n$-fold rotational symmetry the beam split into $k n$ first-order $m=\operatorname{sign}(m) 1$ vortices arranged symmetrically around one $\operatorname{sign}(m)(|m|-k n)$ vortex with $k$ equaling the down-rounded $m / n$. Consequently, if $m<n$, the $m$-order beam is protected from splitting. For example, an $m=4$ beam starts to split into four off-center $m=1$ when centered at the fourfold symmetric TiO column [Fig. 5(a)], whereas the $m=2$ beam stays stable [Fig. 5(b)]. Similarly an $m=6$ beam splits into four off-center $m=1$ and one centered $m=2$ beam. We emphasize, however, that perfect $n$-fold rotational site symmetry is never fulfilled in practice, due to, e.g., a nonperfect centering of the beam, imperfect apertures, or the thermal motion of the atoms. In practice, these perturbations lead to the splitting of all high-order beams into first-order beams and we observe (up to $m=6$ ) the following modified splitting for an $m$ vortex on an approximately $n$-fold symmetric site into $k n$ symmetric off-center $m=\operatorname{sign}(m) 1$ vortices and $|m-k n|$ center $m=\operatorname{sign}(m-k n) 1$ vortices
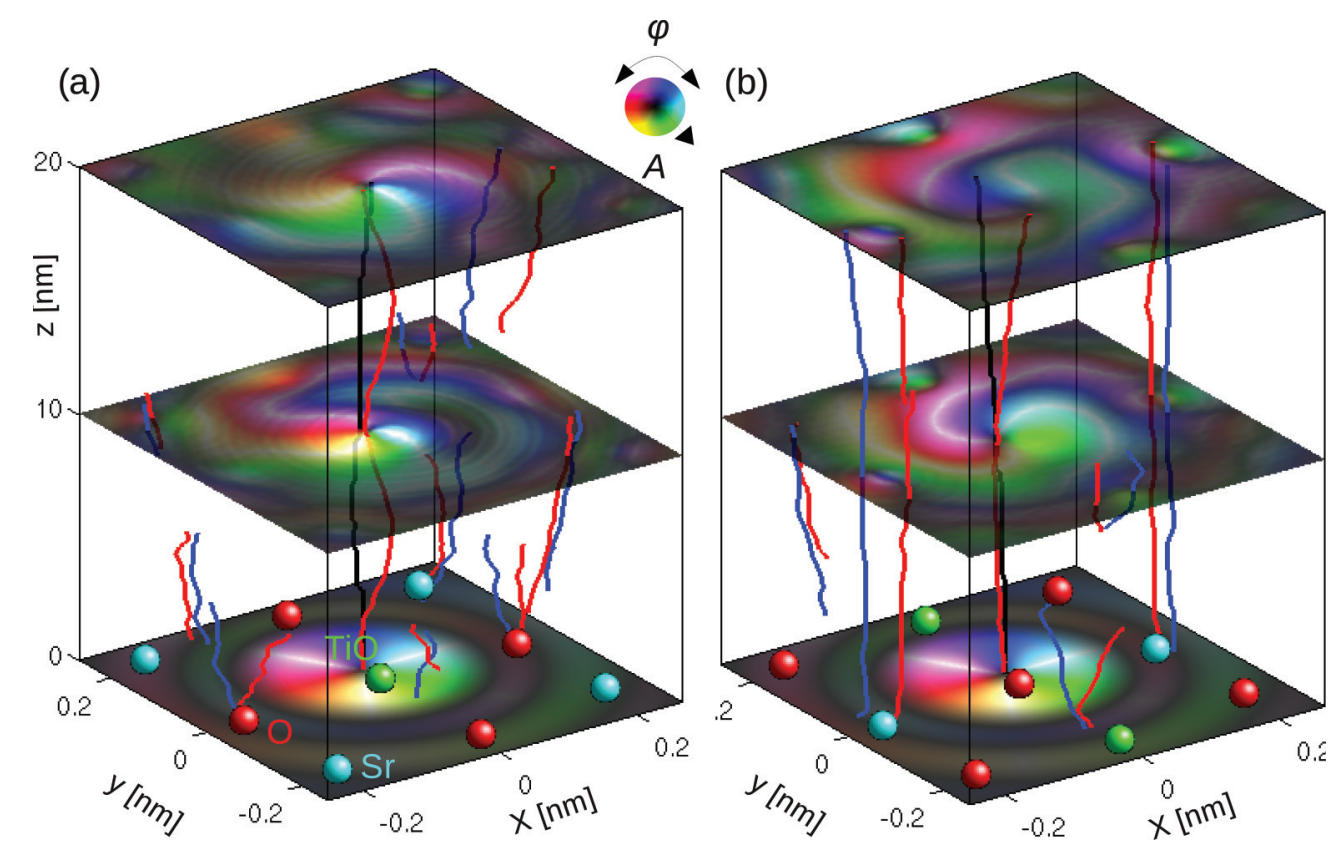

FIG. 4. (Color online) $m=1$ vortex beam slightly off-center (a) the $\mathrm{TiO}$ and (b) the $\mathrm{O}$ column. The wave functions at different $z$ planes are displayed domain colored. $m=1(-1)$ vortex lines are blue (dark gray) [red (light gray)]. Center-of-mass line is black. 


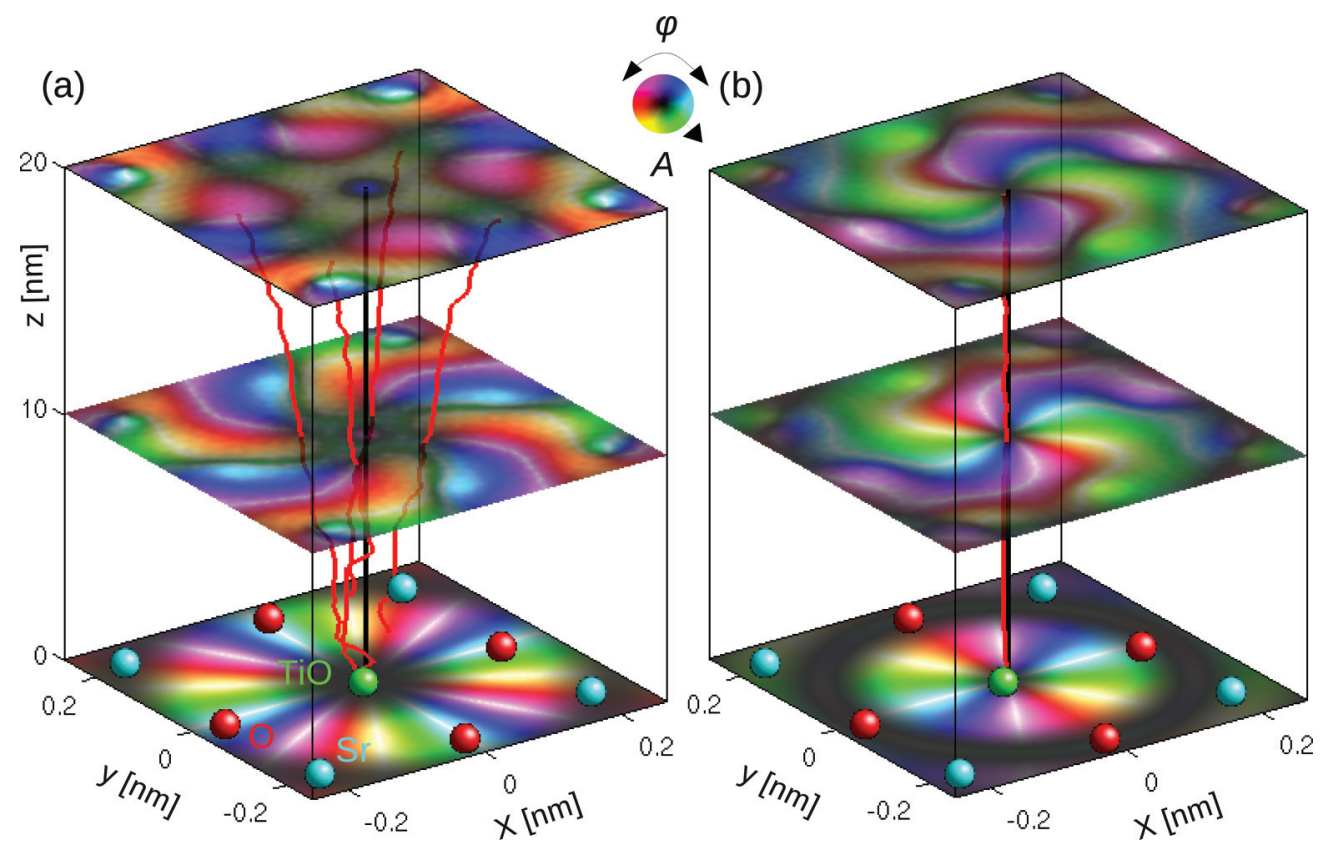

FIG. 5. (Color online) (a) $m=4$ and (b) $m=2$ vortex beams centered at the TiO column. The wave functions at different $z$ planes are displayed domain colored. $m=1(-1)$ vortex lines are blue (dark gray) [red (light gray)]. Center-of-mass line is black.

with

$$
k=\left\{\begin{array}{lll}
\lfloor|m| / n\rfloor & \text { if } & \bmod (|m|, n) \leqslant n / 2 \\
\lceil|m| / n\rceil & \text { if } & \bmod (|m|, n) \geqslant n / 2
\end{array}\right.
$$

Here, $\lfloor\cdot\rfloor(\lceil\cdot\rceil)$ denote rounding down (up). For example, the $m=3$ centered on the fourfold symmetric TiO column, protected under perfect symmetry, now splits into four offcenter $m=1$ and one centered $m=-1$ vortex. Similarly, additional vortex-antivortex pairs with a symmetry-adapted distribution may occur in the wave field (not shown in Fig. 5).

\section{SUMMARY AND DISCUSSION}

We have analyzed the fabric of vortex lines occurring in a vortex beam upon propagation through an atomic lattice with respect to general topological information. The following features with corresponding significance could be distinguished: (i) a Rankine-like vortex structure with significantly reduced diffusion of the vortex beam compared to a nonvortex beam, when centered on an atomic column. Therefore vortex beams hold the promise to significantly improve atomic resolution STEM-EELS techniques, where the ubiquitous delocalization of the probe upon propagation systematically obscures the atomic information [26]; (ii) a systematic deflection of vortex lines from amplitude and phase perturbations leading, e.g., to a circulation of vortex lines around atomic columns depending on the sign of the vorticity; (iii) symmetry-dependent splitting of $m$-order vortex beams. We showed that combining topological concepts (conservation of total winding number) and symmetry allows predicting the vortex line structure of the beam without solving the scattering equations at all. In particular, one can predict which high-order vortex beam will maintain a centered topologically protected zero under certain rotational symmetries; and (iv) the appearance of vortex-antivortex pairs corresponding to not-knotted vortex loops predominantly attached to atomic columns. That observation is important for the design of phase unwrapping algorithms for electron holography, where the linking of phase singularities represents the major obstacle [27]. We furthermore point out that the three-dimensional vortex line behavior is useful to classify vortex beam creators in general. The closeness of vortex lines implies that in order to produce a vortex one has to create a vortex-antivortex pair in the first place and before singling out, later on, one of these. This can be done in a symmetric fashion, e.g., fork [1] and spiral [6] aperture or in an unsymmetrical fashion, e.g., the finite thickness phase plate [2].

\section{ACKNOWLEDGMENTS}

The authors acknowledge funding from the European Research Council under the 7th Framework Program (FP7), ERC Grant No. 246791 - COUNTATOMS and ERC Starting Grant No. 278510- VORTEX. Financial support from the European Union under the 7th Framework Program under a contract for an Integrated Infrastructure Initiative No. 312483ESTEEM2 is also acknowledged.

\section{APPENDIX A: WINDING NUMBER, VORTICITY, ANGULAR MOMENTUM}

In the following we note some definitions partly utilized in the text and frequently used in the context of vortex physics. The winding number can be obtained from various definitions

$2 \pi w \equiv \oint \nabla \varphi \cdot d s=-i \oint \frac{\nabla \Psi}{\Psi} \cdot d s=\iint_{S} \nabla \times \nabla \varphi \cdot n d^{2} r$,

where the kernel of the last surface integral defines the phase vorticity distribution

$$
\boldsymbol{\Theta}_{p} \equiv \nabla \times \nabla \varphi
$$


used in the text. The vorticity is defined in the following way:

$$
\begin{aligned}
\boldsymbol{\Theta} & \equiv \nabla \times \boldsymbol{v}=\frac{1}{m} \nabla \times j \\
& =\frac{-i \hbar}{2 m^{2}} \nabla \times\left(\Psi^{*} \nabla \Psi-\Psi \nabla \Psi^{*}\right) \\
& =\frac{-i \hbar}{m^{2}} \nabla \Psi^{*} \times \nabla \Psi=\frac{2 \hbar}{m^{2}} \nabla \operatorname{Re} \times \nabla \mathrm{Im} \\
& =\frac{-i \hbar}{m^{2}} \nabla \times(\rho \nabla \varphi) \\
& =\frac{-i \hbar}{m^{2}}(\nabla \rho \times \nabla \varphi+\rho \nabla \times \nabla \varphi) .
\end{aligned}
$$

The last line demonstrated that vorticity should not be confused with the phase vorticity distribution. The angular momentum density reads

$$
\boldsymbol{L}=m \boldsymbol{r} \times \boldsymbol{j}=\frac{-i \hbar}{2} \boldsymbol{r} \times\left(\Psi^{*} \nabla \Psi-\Psi \nabla \Psi^{*}\right)
$$

and is only directly proportional to the vorticity in the case of an angular momentum eigenstate.

\section{APPENDIX B: PARAXIAL EQUATION IN A ROTATING BASIS}

The paraxial wave equation in a rotated basis used in the text [Eq. (8)] is found by performing the following substitution in the standard paraxial equation: $\Phi(\boldsymbol{r})=\chi(\boldsymbol{r}) \exp (\operatorname{im} \theta)$,

$$
\begin{aligned}
\partial_{z} \Phi(\boldsymbol{r})= & i\left(\frac{E V(\boldsymbol{r})}{k_{0 z} \hbar^{2} c^{2}}+\frac{1}{2 k_{0 z}} \Delta_{\perp}\right) \Phi(\boldsymbol{r}) \\
= & i\left[\frac{E V(\boldsymbol{r})}{k_{0 z} \hbar^{2} c^{2}} \Phi(\boldsymbol{r})+\frac{\exp (i m \theta)}{2 k_{0 z}}\left(\Delta_{\perp} \chi(\boldsymbol{r})\right.\right. \\
& \left.\left.-\frac{m^{2}}{r_{\perp}^{2}}+\frac{2 i m}{r_{\perp}^{2}}\left(x \partial_{y}-y \partial_{x}\right) \chi(\boldsymbol{r})\right)\right]
\end{aligned}
$$

which leads to the following differential equation for the nonrotating $\chi$ :

$\partial_{z} \chi(\boldsymbol{r})=i\left(\frac{E V(\boldsymbol{r})}{k_{0 z} \hbar^{2} c^{2}}+\frac{1}{2 k_{0 z}} \Delta_{\perp}-\frac{m^{2}}{2 k_{0 z} r_{\perp}^{2}}+\frac{i m}{k_{0 z} \hbar r_{\perp}^{2}} \hat{L}_{z}\right) \chi(\boldsymbol{r})$.

The last term in the parentheses is missing for OAM eigenstates because there $\chi(\boldsymbol{r})=\chi\left(r_{\perp}, z\right)$.

\section{APPENDIX C: ANALYTIC EXPRESSION FOR PARAXIAL VORTEX BEAMS}

To calculate the initial shape of the vortex beam at the sample plane formed in the microscope equipped with a fork aperture we need to consider first the aperture plane (radius $Q$ ) and subsequently propagate the solution to the specimen plane. In the fork aperture plane $\Psi=\Theta(q-Q) \mid \exp \left(i \frac{k_{0}}{2} q\right)+$ $\left.\exp \left(-i \frac{k_{0}}{2} q\right) \exp (i m \varphi)\right|^{2}=\Theta(q-Q)\left[2+\exp \left(i k_{0} q\right)\right.$ $\exp (-i m \varphi)+$ c.c.] assuming a nonbinary fork aperture (binarization mainly introduces higher-order vortices, which we neglect here). The wave function in the specimen plane, obtained by Fourier transformation, consists of an Airy-shaped center band $(m=0)$, and two complex conjugate sidebands $( \pm m)$ with

$$
\begin{aligned}
\Psi_{m}\left(\boldsymbol{r}_{\perp}\right)= & \int d^{2} q \Theta(q-Q) \exp (i m \varphi) \exp \left(i \boldsymbol{q} \boldsymbol{r}_{\perp}\right) \\
= & \int_{0}^{Q} \int_{0}^{2 \pi} d q d \varphi q \exp (i m \varphi) \exp [i q r \cos (\theta-\varphi)] \\
= & 2 \pi i^{m} \exp (i m \theta) \int_{0}^{Q} d q q J_{m}\left(q r_{\perp}\right) \\
= & \exp (i m \theta) \frac{2 \pi i^{m} 2^{-m} r_{\perp}^{m} Q^{2+m}}{(2+m) \Gamma(1+m)} \\
& \times{ }_{1} F_{2}\left(1+\frac{m}{2} ; 2+\frac{m}{2}, 1+m ;-\frac{1}{4} r_{\perp}^{2} Q^{2}\right), \quad(\mathrm{C} 1)
\end{aligned}
$$

where ${ }_{p} F_{q}(a ; b ; z)$ is the generalized hypergeometric function [28]. Accordingly, a vortex beam with the characteristic phase $\exp (\operatorname{im} \theta)$ is obtained by cutting out one sideband. It has to be noted, however, that the support of ${ }_{1} F_{2}(a ; b ; z)$ is not limited, i.e., the sidebands reach into the center band and vice versa. That effect and the binary character of typical fork apertures lead to additional modifications to $\Psi_{m}$, which will be neglected here. For the relevant cases of $m=1,2,4$ used in this work the expression simplifies to

$$
\begin{gathered}
\Phi_{m=1}\left(\boldsymbol{r}_{\perp}\right)=\frac{i \pi^{2} k_{\max }}{r_{\perp}} \exp (\operatorname{im} \theta)\left[J_{1}\left(k_{\max } r_{\perp}\right) H_{0}\left(k_{\max } r_{\perp}\right)\right. \\
\left.-J_{0}\left(k_{\max } r_{\perp}\right) H_{1}\left(k_{\max } r_{\perp}\right)\right], \\
\Phi_{m=2}\left(\boldsymbol{r}_{\perp}\right)=\frac{-2 \pi}{r_{\perp}^{2}} \exp (i m \theta)\left[2-2 J_{0}\left(k_{\max } r_{\perp}\right)\right. \\
\left.-Q r_{\perp} J_{1}\left(k_{\max } r_{\perp}\right)\right]
\end{gathered}
$$

and

$$
\Phi_{m=4}\left(\boldsymbol{r}_{\perp}\right)=\exp (\operatorname{im} \theta) \frac{4 r_{\perp}+\left(r_{\perp}^{2} k_{\max }-\frac{8}{k_{\max }}\right) J_{1}\left(k_{\max } r_{\perp}\right)-8 r_{\perp} J_{2}\left(k_{\max } r_{\perp}\right)}{r_{\perp}^{3}},
$$

where $J$ denotes Bessel functions of the first kind and $H$ denotes Struve functions.

[1] J. Verbeeck, H. Tian, and P. Schattschneider, Nature (London) 467, 301 (2010).

[2] M. Uchida and A. Tonomura, Nature (London) 464, 737 (2010).

[3] K. Y. Bliokh, Y. P. Bliokh, S. Savel'ev, and F. Nori, Phys. Rev. Lett. 99, 190404 (2007).
[4] G. Molina-Terriza, J. P. Torres, and L. Torner, Nat. Phys. 3, 305 (2007).

[5] S. Franke-Arnold, L. Allen, and M. Padgett, Laser \& Photonics Rev. 2, 299 (2008).

[6] J. Verbeeck, H. Tian, and A. Béché, Ultramicroscopy 113, 83 (2012). 
[7] J. Verbeeck, P. Schattschneider, S. Lazar, M. Stoger-Pollach, S. Loffler, A. Steiger-Thirsfeld, and G. V. Tendeloo, Appl. Phys. Lett. 99, 203109 (2011).

[8] S. Lloyd, M. Babiker, and J. Yuan, Phys. Rev. Lett. 108, 074802 (2012).

[9] P. Schattschneider, M. Stöger-Pollach, and J. Verbeeck, Phys. Rev. Lett. 109, 084801 (2012).

[10] P. Schattschneider and J. Verbeeck, Ultramicroscopy 111, 1461 (2011).

[11] K. Y. Bliokh, P. Schattschneider, J. Verbeeck, and F. Nori, Phys. Rev. X 2, 041011 (2012).

[12] M. V. Berry, J. Opt. A: Pure and Appl. Opt. 11, 094001 (2009).

[13] M. V. Berry and M. R. Dennis, J. Phys. A: Math. Theor. 40, 65 (2007).

[14] K. Poelke and K. Polthier, Comput. Graph. Forum 28, 735 (2009).

[15] T. Frankel, The Geometry of Physics: An Introduction (Cambridge University Press, Cambridge, 1999).

[16] I. Freund, Opt. Commun. 159, 99 (1999).

[17] M. J. Lighthill, An Informal Introduction to Theoretical Fluid Mechanics (Oxford University Press, New York, 1986).
[18] M. D. Graef, Introduction to Conventional Transmission Electron Microscopy (Cambridge University Press, Cambridge, 2003).

[19] S. Löffler and P. Schattschneider, Acta Crystallogr. Sect. A: Found. Crystallogr. 68, 443 (2012).

[20] J. Cowley and A. Moodie, Acta Crystallogr. 10, 609 (1957).

[21] D. Rozas, C. T. Law, and J. G. A. Swartzlander, J. Opt. Soc. Am. B 14, 3054 (1997).

[22] A. Weickenmeier and H. Kohl, Acta Crystallogr. Sect. A: Found. Crystallogr. 47, 590 (1991).

[23] D. J. Acheson, Elementary Fluid Dynamics (Oxford University Press, New York, 1990).

[24] L. Allen, H. Faulkner, M. Oxley, and D. Paganin, Ultramicroscopy 88, 85 (2001).

[25] J. Leach, M. R. Dennis, J. Courtial, and M. J. Padgett, Nature (London) 432, 165 (2004).

[26] R. Egerton, Electron Energy-Loss Spectroscopy in the Electron Microscope (Plenum Press, New York, 1996).

[27] D. C. Ghiglia and M. D. Pritt, Two-Dimensional Phase Unwrapping (Wiley, New York, 1998).

[28] M. Abramowitz and I. A. Stegun, Handbook of Mathematical Functions with Formulas, Graphs, and Mathematical Tables (Dover, New York, 1964). 